\title{
Chemical Induction of Human Adipose Stromal Cells Into Hepatocyte-Like Cells under Various Differentiation Conditions
}

\section{Coronado Ramon ${ }^{1 *}$, Somaraki-Cormier Maria ${ }^{1}$, Natesan Shanmugasundaram², Christy Robert ${ }^{2}$, Ong Joo ${ }^{3}$ and Halff Glenn ${ }^{4}$}

${ }^{1}$ Lester Smith Medical Research Institute, San Antonio, TX, USA

2US Army Institute of Surgical Research, JBSA Fort Sam Houston, TX, USA

${ }^{3}$ University of Texas at San Antonio, San Antonio, TX, USA

${ }^{4} U T$ Health San Antonio, San Antonio, TX, USA

\begin{abstract}
Background: A shortage of donor livers for transplant has led to an increased interest in cell therapeutics as an alternative to whole organ transplant to treat end-stage liver disease. Primary human hepatocytes have been used in cell-based therapies. However, hepatocytes do not proliferate in vitro so it is challenging to grow enough cells for a successful transplant. Many have suggested using hepatocyte-like Adipose-derived mesenchymal Stromal/ stem Cells (ASCs) differentiated into hepatocyte-like cells as a substitute. Here we evaluate how closely these cells resemble primary hepatocyte cell morphology and function.
\end{abstract}

Methods: Human ASCs were mechanically isolated from lipoaspirates. The stem cell nature of ASCs was characterized using flow cytometry and tri-lineage differentiation into osteocytes, adipocytes, and chondrocytes. ASCs were differentiated into hepatocyte-like cells in culture using various protocols that included combinations of growth factors and small molecules. Primary ASCs quickly attached and proliferated in vitro, forming a homogeneous spindle-like cell monolayer. Mesenchymal stem cells showed high expression of the markers CD73, CD90, CD271, CD44, CD166, CD105, and successfully differentiated into osteocytes, chondrocytes, and adipocytes. ASCs were cultured on type I collagen coated plates and differentiated into hepatocyte-like cells using 5 different protocols.

Results: The ASCs differentiated into hepatocyte-like cells, using protocol C (induction with FGF4 and maturation with HGF, ITSPre, Dex, OncM and $2 \%$ serum), displayed a cuboidal morphology. Bioactivity assays demonstrated their ability to synthesize urea, uptake LDL, and metabolize glucose; all cardinal characteristics of hepatocytes, not present in undifferentiated ASCs. Gene expression analysis also showed the expression of several genes known to play an important role in liver function including, TDO2, ALB, HNF1B1, HNF6b, HNF4a, and AFP. However, even the best hepatocyte-like induced ASC obtained in this study had much inferior hepatocyte-related gene expression levels compared to primary human hepatocytes.

Conclusion: We successfully differentiated ASCs into hepatocyte-like cells; Protocol C produced the best hepatocyte-like cells based on morphology and function typically seen in primary hepatocytes. Although the results displayed some hepatocyte-related function, comparison of bioactivity and gene expression of hepatocyte-like cells were drastically lower than those of primary human hepatocytes, suggesting that caution should be taken when considering using differentiated hepatocyte-like ASCs to replace hepatocytes. Further studies are needed to better understand the functional capacity of hepatocyte-like ASCs, and which specific metabolic function could potentially offer therapeutic applications.

Keywords: Adipose-derived stem cells; Cell-based therapy; Hepatocyte-like cells; Mesenchymal stromal/stem cells; Adipose tissue

\section{Background}

Liver transplantation is a commonly accepted life-saving surgical procedure for a variety of irreversible acute and chronic liver diseases. Advances in the management and surgical techniques have been made since the first human liver transplant [1]. Over the years, this procedure has become routine with an excellent outcome in terms of both quality and extension of life [2,3]. However, the major challenge facing the transplant community, a consequence of their great success, is that more organs for transplantation are now needed. The limited availability of organ donors has led to the search for alternatives to whole-organ transplantation such as cell-based therapies. Hepatocyte transplantations in particular have been successfully performed to treat acute liver failure, end-stage liver disease, and inborn errors of metabolism [4-7]. However, the shortage of organ donors, immune rejection of allogeneic cells, and the fact that primary hepatocytes do not proliferate and lose their function in vitro pose great challenges in obtaining enough high quality primary hepatocytes for clinical treatments $[8,9]$. These difficulties have led to a search for other sources of cells, such as stem cells, that could be substituted for functional hepatocytes [10-13].

Adipose-derived mesenchymal stromal/stem cells have been considered a candidate as a liver progenitor cell source for therapeutic use. Within the mesenchymal stem cell family, ASCs can be isolated from adipose tissue; usually found in excess, thus providing a simple and accessible autologous source of stem cells [14]. In addition, ASCs have

*Corresponding author: Coronado Ramon, Executive Director, Lester Smith Medical Research Institute, 3463 Magic Drive, Suite 315, United States, San Antonio, TX 78229, USA, Tel: 2105859782; E-mail: rcoronado@smithinterests.com

Received April 06, 2018; Accepted April 19, 2018; Published April 30, 2018

Citation: Coronado RE, Somaraki-Cormier M, Christy R, Natesan S, Ong J, et al (2018) Chemical Induction of Human Adipose Stromal Cells Into Hepatocyte-Like Cells under Various Differentiation Conditions. J Stem Cell Res Ther 8: 424. doi: 10.4172/2157-7633.1000424

Copyright: (c) 2018 Coronado RE, et al. This is an open-access article distributed under the terms of the Creative Commons Attribution License, which permits unrestricted use, distribution, and reproduction in any medium, provided the original author and source are credited. 
lower therapeutic risk associated with immune rejection and teratoma formation compared to other types of stem cells like induced Pluripotent Stem Cells [15-20]. In culture, ASCs can be expanded without losing their stemness, compared to the non-proliferative hepatocytes, leading to the advantage of obtaining high cell yields capable of differentiating into hepatocyte-like cells [21]. ASCs from different origins and species can be chemically induced to differentiate into hepatocyte-like cells that demonstrate bioactivity and morphology otherwise exclusive to that of native hepatocytes [22-28]. Despite published results showing hepatocyte-like cells derived from differentiated ASCs, only a limited group of unique molecules/functions are shown to be up-regulated compared to the plethora of molecules/functions expressed in true hepatocytes [13,22,24,25,29-31].

There have been extensive efforts to improve the conditions in which hepatocyte and hepatocyte-like cells can be cultured in vitro $[32,33]$. These efforts include optimizing the culturing conditions, by the addition of cytokines, use of various media, coatings, gels, and sandwich methods, as well as 2-dimension (2-D) or 3-dimension (3D) culturing conditions [34-40]. More recent approaches in hepatocyte culture conditions have included mimicking the native physiological niche where hepatocytes are found, with the aim to emulate the proteins and signals found in vivo [38,39,41]. However, the ideal combination of different proteins as well as different cocktails of cytokines for optimal culture conditions are yet to be achieved [37]. Of the currently available matrices, such as type I collagen, fibronectin, and matrigel, none has demonstrated long-term success [34,36,39,42-46]. Better differentiation of ASCs into hepatocyte-like cells will facilitate long-term functional hepatic integration when applied in vivo [27]. Our goal is to optimize the culture conditions for the differentiation of ASCs into functional hepatocyte-like cells in vitro.

\section{Materials and Methods}

\section{Mechanical isolation of adipose derived mesenchymal stromal/stem cells}

Adipose tissues obtained from human liposuction were processed using Lipogems ${ }^{\oplus}$, which is a class II, single-use medical device for the processing of aspirated adipose tissue. The unit consists of a transparent plastic cylinder with filters and beads for the micro fracturing of adipose tissue. Lipoaspirates from healthy individuals donated by Dr. Lindsey Coombs were received and added into the Lipogems device. Permission for the collection of tissue and subsequent research was unnecessary in Los Angeles because fat was collected under an institutional review board exemption as medical waste. The device further processed the lipoaspirate by washing and mechanically disrupting it into micronized adipose tissue. The resulting micronized adipose tissue was collected and seeded in tissue culture treated plastic-ware at proportions of $2 \mathrm{~mL}$ per $25 \mathrm{~cm}^{2}$ and placed in a $5 \% \mathrm{CO}_{2}$ humidified incubator at $37^{\circ} \mathrm{C}$ for 3 hours, then $3 \mathrm{~mL}$ of Mesempro RS ${ }^{\oplus}$ media was added. Fresh media was added $(3 \mathrm{~mL})$ every 3 days without replacing the old media until day 9. On day 9 a total media replacement was performed without disturbing the attached pieces of micro-fat. On day 12 , complete media replacement was repeated after removing non-adhered pieces of microfat leaving only attached ASCs in the flask. The cells were trypsinized (0.25\% Trypsin-EDTA Gibco Cat.\#25200056) when they reached confluency, washed, and sub-cultured for further analysis.

\section{Characterization of ASCs by flow cytometry}

Collected cells were washed in running buffer (MACS Cat. \#130091-221) and centrifuged at $200 \times \mathrm{g}, 4^{\circ} \mathrm{C}$, for 5 minutes and re-suspended in BlockAid (Thermo Cat. \# B10710) $\times 10$ for a final concentration of 15 cells/test $/ 100 \mu \mathrm{L}$. Cells were incubated in BlockAid for 15 minutes at $4^{\circ} \mathrm{C}$, followed by addition of primary-conjugated antibodies according to manufacturer's instructions, and incubated at $4^{\circ} \mathrm{C}$, in the dark, for 1 hour. Stained samples were washed with $1 \mathrm{~mL}$ running buffer $(200$ $\times \mathrm{g}$ for 5 minutes at $4^{\circ} \mathrm{C}$ ) twice; then reconstituted in $200 \mu \mathrm{L} / \mathrm{sample}$ running buffer before measurements were taken. Flow cytometry analysis was performed with a CytoFLEX 2-L (Beckman-Coulter). Commercially available adipose derived stem cells from lipoaspirates (Thermo Cat. \# R7788115) were used as positive controls. Results were quantitated by CytExpert software (Beckman-Coulter). Antibodies used for flow cytometry: Anti-HLA-DR-FITC-Class II (Molecular Probes \#MHLDR01), CD44-FITC (Thermo \#MEM-85), CD271VioBright FITC (MACS \#130-110-115), CD166-PE (MACS \#130-106618), CD105-PE (StemCell Technologies \#60039PE), CD90-PerCPCy5.5 (Molecular Probes \#A16425), CD19-PE (StemCell Technologies \#60005PE), CD11b-PE (StemCell Technologies \#60040PE), CD34-PE (StemCell Technologies \#60013PE), CD45-PE (StemCell Technologies \#60018PE), CD73-PE (StemCell Technologies \#60044PE).

\section{Tri-lineage differentiation}

Multipotency of isolated mesenchymal stem cells from adipose tissue aspirates was confirmed by tri-lineage differentiation (i.e., adipocyte, osteocytes, and chondrocytes), phenotypic expression, and by flow cytometry. In order to differentiate mesenchymal stem cells into chondrocytes, it was necessary to generate micromass cultures, by seeding $5-\mu \mathrm{L}$ droplets $\times 10$ of cell solution $(1.67$ cells $/ \mathrm{mL})$ in a 6 well plate (approximately 10-15 droplets per well) and culturing for 2 hours under standard culture conditions. Prewarmed StemPro ${ }^{\circ}$ Chondrogenesis Differentiation Kit (Thermo Cat.\#A1007101) media was added to the culture vessels and incubated at $37^{\circ} \mathrm{C}, 5 \%$ $\mathrm{CO}_{2}$. Cells for adipose and osteogenic differentiation were seeded in 6-well plates at $10 \times 103 \mathrm{cells} / \mathrm{cm}^{2}$ and incubated at $37^{\circ} \mathrm{C}, 5 \% \mathrm{CO}_{2}$ in MesenPRO RS ${ }^{\mathrm{mx}}$ Basal Medium supplemented with MesenPRO RS ${ }^{\mathrm{mm}}$ Growth Supplement (Gibco Cat.\#12746012), antibiotic-antimycotic (Thermo Cat.\#15240062), and Glutamax (Thermo Cat.\#35050061), until they reached $80 \%$ confluency, then the pertinent differentiation media was added, StemPro ${ }^{\circ}$ Adipogenesis Differentiation Kit (Thermo Cat.\#A1007001) and StemPro ${ }^{\circ}$ Osteogenesis Differentiation Kit (Thermo Cat.\#A1007201), respectively. The differentiation media were replaced every 2 days according to manufacturer's instructions. Alizarin Red S (Sigma Cat.\#A5533), Oil Red O (Sigma Cat.\#O1391) and Alcian Blue (Sigma Cat.\#B8438) staining were used to confirm presence of the adipose-derived osteocytes, adipocytes, and chondrocytes, respectively. Specifically, calcium deposition by adipose-derived osteocytes was visualized by Alizarin Red staining or Van Kossa staining. Cells were fixed with $4 \%$ buffered formaldehyde for 30 minutes and washed twice with distilled deionized water. When stained with Alizarin Red S, the cells were treated with a $0.5 \mathrm{M} \mathrm{pH} 4.2$ Alizarin Red solutions for 2-3 $\mathrm{min}$, then washed again with distilled deionized water. Von Kossa stain was performed as follows: $1 \%$ silver nitrate solution was added to the cells and the plates were placed on a transluminator to be irradiated with UV light for 1-2 h. Each well was washed with distilled deionized water and then treated with $5 \%$ sodium thiosulfate for $1 \mathrm{~min}$, followed by several rinses with distilled deionized water. Proteoglycan production by adipose-derived chondrocytes was visualized by Alcian blue. All cells were fixed with $4 \%$ buffered formaldehyde for 30 minutes, washed twice with distilled deionized water, then stained with $1 \%$ Alcian Blue in 3\% Acetic Acid for 1-2 hrs at room temperature, followed by the addition of $0.1 \mathrm{~N} \mathrm{HCl}$ solution for 30 minutes, then three rinses with distilled deionized water to neutralize the acid. Chondrogenic pellets 
were fixed in $4 \%$ buffered formaldehyde, dehydrated through graded alcohol washes $(70,95$, and $100 \%)$, cleared with xylene (EMD, Millipore, USA), and embedded in paraffin. The sectioned $(10 \mu \mathrm{m}$ thick) chondrogenic pellets were placed on glass slides, then de-waxed to undergo Alcian Blue staining. Lastly, oil production (droplets) in ASC-derived adipocytes was visualized by Oil Red O staining. Briefly, cells were fixed with $4 \%$ buffered formaldehyde for 30 minutes, washed twice with distilled deionized water, then stained with Oil red O $(0.5 \%$ in isopropanol) diluted with water (3:2) and incubated for 15 minutes at room temperature. Cells were then washed with water and the stained fat droplets were visualized by phase-light microscopy.

\section{Differentiation of ASCs into hepatocyte-like cells}

ASCs were cultured on type I collagen substrate and differentiated into hypatocyte-like cells using five different chemically induced differentiation protocols. ASCs were seeded in a 6 well plate $\left(5 \times 10^{5}\right.$ cells/well) pre-coated with type I collagen (Rat Collagen I Coated Plates Gibco $^{\oplus}$ Cat.\#A1142801) optimized for hepatocyte culture according to manufacturer's instructions. ASCs were cultured at $37^{\circ} \mathrm{C}, 5 \% \mathrm{CO}_{2}$ in MesenPRO RS ${ }^{\text {ma }}$ Basal Medium supplemented with MesenPRO RS $^{\text {ma }}$ Growth Supplement (Gibco Cat.\#12746012), antibiotic-antimycotic (Thermo Cat.\#15240062), and Glutamax (Thermo Cat.\#35050061), until they reached $80 \%$ confluency. The ASC media was then changed to serum-free low glucose DMEM (Gibco Cat.\#10567) plus antibiotics for $24 \mathrm{hrs}$ (pre-induction step), followed by their corresponding protocol, see (Table 1). The concentration of each growth factor was: 20 ng/mL human recombinant Epidermal Growth Factor (EGF Gibco Cat.\#PHG0311), $10 \mathrm{ng} / \mathrm{mL}$ basic Fibroblast Growth Factor (bFGF Gibco Cat.\#13256029, 10 ng/mL Fibroblast Growth Factor (FGF4 Gibco Cat.\#PHG0154), $20 \mathrm{ng} / \mathrm{mL}$ Hepatocyte Growth Factor (HGF Gibco Cat.\# PHG0324), 0.61 g/L nicotinamide (Sigma Cat.\#N0636), $20 \mathrm{ng} / \mathrm{mL}$ OncostatinM (OSM Gibco Cat.\#PHC5015), $1 \mathrm{mmol} / \mathrm{L}$ Dexamethasone (Dex) (AlfaAesar Cat.\#A17590), 50 mg/mL InsulinTransferrin-Selenium (ITS Gibco Cat.\#41400045), 2\% Knockout Replacement Serum (Gibco Cat.\#10828028), $\mu$ MPalmitoyl20ng/ mLcoenzymeActivin A (Thermo Cat.\#PHC9564), 2 lithium salt $\mu \mathrm{M}$ Tr (Sigmacostatin Cat.A\#(TSAP9716), Sigma1 Cat.\#T8552).

\section{Urea synthesis}

Urea secretion was measured by collecting the supernatant from the cultured cells at various time points and using a colorimetric assay kit (QuantiChromTM Urea Assay Kit) according to the manufacturer's instructions. Known concentrations of urea were tested in parallel with the experimental samples and all of the measurements were performed in a Synergy HTX Multi-Mode Reader (Biotek ${ }^{\circledR}$, VT, USA). Sample values were normalized by cell number, quantified by MTT.

\section{Cytochrome P450 (C3A4) metabolism}

Cytochrome P450 (CYP3A4) was measured at the end of the hepatocyte-differentiation protocol using a P450-GloTM CYP3A4 Assay with Luciferin- isopropyl acetal (IPA, Promega). After incubation, an aliquot of $50 \mu \mathrm{L}$ medium was transferred from each well to a 96well opaque white luminometer plate at room temperature. Then, luciferin detection reagent $(50 \mu \mathrm{L})$ was added to each well and the plate was allowed to stand for $20 \mathrm{~min}$ in the dark to initiate a luminescent reaction. The resulting luminescence was read using a Synergy HTX Multi-Mode reader (Biotek ${ }^{\oplus}$, VT, USA). Sample values were normalized by cell number, quantified by MTT.

\section{Periodic Acid-Schiff (PAS)}

Glycogen storage of cultured hepatocytes was evaluated by Periodic Acid-Schiff (PAS) staining. Cells in culture were fixed in $4 \%$ buffered formaldehyde (Fisher Scientist Cat.\#F79-4) for 15 minutes, then treated with $1 \%$ periodic acid solution for 5 minutes at room temperature, rinsed three times in distilled deionized water and exposed to Schiff's reagent for 15 minutes for subsequent color development. Finally, wells were washed with distilled deionized water for 5-10 min and observed by bright-field microscopy (EVOS FL, Life Technologies).

\section{Uptake of low-density lipoprotein}

The uptake of low-density lipoprotein (LDL) was detected with the Dil-Ac-LDL staining kit (Biomedical Technologies, Stoughton, MA, USA) according to the manufacturer's instructions. ASCs and hepatocyte-like cells were incubated in serum-free DMEMLG containing $10 \mu \mathrm{g} / \mathrm{mL} \quad 1,1^{\prime}$-dioctadecyl-3,3,3',3'-tetramethylindocarbocyanine perchlorate acetylated-LDL (Dil-Ac-LDL) for $4 \mathrm{~h}$ at $37^{\circ} \mathrm{C}$. Cells were then washed and visualized under a fluorescence microscope (EVOS FL, Life Technologies).

\section{Viability and proliferation}

An MTT (3-(4, 5-dimethylthiozole-2-yl)-2, 5-diphenyltetrazolium bromide, Molecular Probes M6494) assay was used to evaluate cell number/viability. A standard curve with known cell number was used at each time point evaluated $5 \mathrm{MTTmg} / \mathrm{mL}$ )solutionwas added ( 25 to each well and incubated for $5 \mathrm{hrs}$ in a $5 \% \mathrm{CO}_{2}$, at $37^{\circ} \mathrm{C}$, humidified incubator. After incubation, the formazan complex was extracted with DMSO and the absorbance was measured at $570 \mathrm{~nm}$.

\section{Characterization and Gene Analysis of Hepatocyte-like Cells by RT-PCR}

To confirm gene expression and function of ASCs-derived hepatocyte-like cells, we studied the expression of genes specific to key regulatory functions, metabolic, nuclear transcription, and protein expression of hepatocytes/hepatoblasts. Total ribonucleic acid (RNA), from cells cultured in 6 well plates, was isolated using TRIzol ${ }^{\circ}$ LS Reagent (Thermo Cat. \#10296010) according to manufacturer's instructions for real-time polymerase chain reaction. The cells were rinsed with HBSS and carefully scrapped while adding $2 \mathrm{~mL}$ of TRIzol ${ }^{\circledR}$ LS Reagent, followed by incubation on ice for $15 \mathrm{~min}$. To separate the aqueous from the solvent phase $1 \mathrm{~mL}$ of chloroform was added to each sample and mixed well followed by centrifugation $(13,000 \times \mathrm{g})$. Total RNA was purified using Qiagen’s mini spin columns (Qiagen Cat. \#27115). The concentration and quality of the purified RNA was determined at 260/280-wavelength optical density ratio using a NanoDrop spectrometer (NanoDrop Technologies, Inc., Wilmington, DE, USA). Complementary deoxyribonucleic acid (cDNA) was synthesized from $150 \mathrm{ng}$ of total RNA using SuperScript III first-strand synthesis supermix with oligo-dT primers (Invitrogen Cat. \#11752250). A negative control sample $\left(\mathrm{H}_{2} \mathrm{O}\right)$ was used to assess random production of cDNA through contaminants. Oligonucleotide primer sequences were designed using Invitrogen's OligoPerfectTM Designer and National Center for Biotechnology Information (NCBI) gene database and manufactured by Thermo- Invitrogen Custom DNA Oligos for the following hepatocyte-specific genes; Alphafetoprotein (AFP), Coagulation Factor IX (F9), G-glutamyltranspeptidase, Hepatocyte Nuclear Factor 4 alpha (HNF4A), Hepatocyte Nuclear Factor 6b (HNF6B), Hepatocyte Nuclear Factor 1 B1 (HNF1B1), phosphoenolpyruvate carboxykinase (PCK), apolipoprotein A-I (APOA1), apolipoprotein C-III (APOC3), 
Citation: Coronado RE, Somaraki-Cormier M, Christy R, Natesan S, Ong J, et al. (2018) Chemical Induction of Human Adipose Stromal Cells Into Hepatocyte-Like Cells under Various Differentiation Conditions. J Stem Cell Res Ther 8: 424. doi: 10.4172/2157-7633.1000424

Page 4 of 10

fibrinogen beta chain (FGB), Glucose-6-phosphatase (G6P), tyrosine aminotransferase (TAT), tryptophan-2,3-dioxygenase (TDO), a-antitrypsin (AAT), albumin (ALB), cytochrome P450/3A4 (C3A4), cytokeratin 18 (CK18), cytokeratin 7 (CK7), forkhead box protein A2 (FOXA2), and Transferrin. Master mixes contained $200 \mathrm{nM}$ of forward and reverse primers containing SYBR Green-ER, and qPCR supermix (Invitrogen Cat.\# 4309155); synthesized cDNA was added to the appropriate wells. Real-time polymerase chain reaction (RT-PCR) was carried out using a Bio-Rad CFX96 thermal cycler system (Bio-Rad, Hercules, CA, USA). Message RNA expression levels were normalized to glyceraldehye-3-phosphate dehydrogenase (GAPDH). Fold increase in expression levels for each specific gene was normalized to the expression levels of control conditions and reported-Ctmethodbythe.

\section{Statistical Analysis}

Numerical data were expressed as the mean SD (standard deviation). Statistical significance was determined using GraphPad Prism 5.00 (GraphPad Software, San Diego California USA). Student's $t$ test was used to determine significant difference between control and test group. Analysis of variance (ANOVA) followed by Newman-Keuls multiple comparison tests was used for analysis of multiple groups, and $\mathrm{p}$ values less than 0.05 were considered statistically significant.

\section{Results}

\section{Characterization of ASCs}

Freshly isolated ASCs quickly adapted in vitro and adhered to the surface of the cell culture plates after the initial seeding. Subsequent days in culture of the ASCs were characterized by rapid cell proliferation forming a homogenous spindle-like cell monolayer (Figure 1a). ASCs showed all required characteristics of multipotent mesenchymal stromal ("stem") cells (MSC) defined by the committee of International Society for Cellular Therapy (ISCT) [47]. First, ASCs adhered to plastic under standard culture conditions. Second, ASCs expressed (>99\%) CD105, CD73, CD90, and CD271, CD44, and CD166 and lacked expression $(<5 \%)$ of the hematopoietic markers; CD45, CD34, CD11b, CD19, and HLA-DR (Figure 1b). Third, ASCs differentiated to osteoblasts, adipocytes and chondrocytes in vitro. Specifically, adipogenic differentiation was demonstrated by the accumulation of neutral lipid (triglycerides) vacuoles determined by the positive Oil Red O staining (Figure 1c). Similarly, chondrocyte aggregates showed generation of a proteoglycan-rich extracellular matrix, a hallmark of chondrogenesis (Figure 1e). Histochemical staining of tissue with Alcian blue was a confirmatory adjunct to immune-localization of collagen type II, a chondrocyte-specific molecule within the cartilage matrix (Figure 1e). In contrast to undifferentiated ASCs, differentiated cells using the osteoblast differentiation protocol presented mineralization, accompanied by the development of nodules that stained positive for phosphate, using Von Kossa staining (Figure 1d) and calcium using and Alizarin Red S staining (data not shown).

\section{Urea Synthesis by hepatocyte-like cells}

To evaluate the efficiency of hepatocyte differentiation exerted by the various protocols (Table 1), we measured urea synthesis, a key function of hepatocytes. Conditioned media from hepatocyte-like cells cultured under the various differentiation conditions showed high levels of urea production only in the conditioned media of cells cultured using protocols $\mathrm{C}, \mathrm{D}$, and $\mathrm{E}\left(\sim 2000-5000 \mathrm{ug} / \mathrm{Dl} / 1 \times 10^{\wedge} 6\right)$ compared to undifferentiated ASCs (control) (Figure 2a).

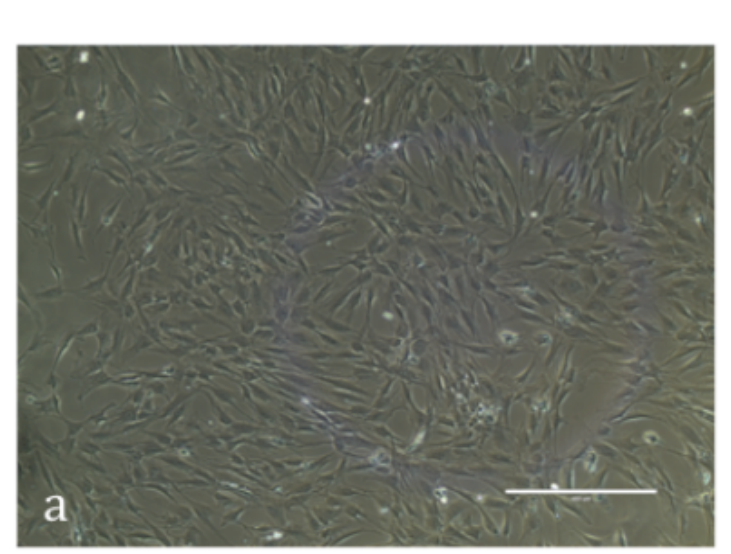

\begin{tabular}{|l|c|c|}
\hline Antibody & POS/NEG & Value \\
\hline CD73 & ++++ & $99.8 \%$ \\
\hline CD90 & ++++ & $99.4 \%$ \\
\hline CD271 & ++++ & $99.3 \%$ \\
\hline CD44 & ++++ & $99.5 \%$ \\
\hline CD166 & ++++ & $99.7 \%$ \\
\hline CD105 & ++++ & $99.9 \%$ \\
\hline CD11b & NEG & \\
\hline CD19 & NEG & \\
\hline CD45 & NEG & \\
\hline CD34 & NEG & \\
\hline HLA-DR & NEG & \\
\hline
\end{tabular}

\begin{tabular}{|l|l|}
\hline$>90 \%$ & ++++ \\
\hline $80-95 \%$ & +++ \\
\hline $30-80 \%$ & ++ \\
\hline $10-30$ & + \\
\hline$<5 \%$ & NEG \\
\hline
\end{tabular}
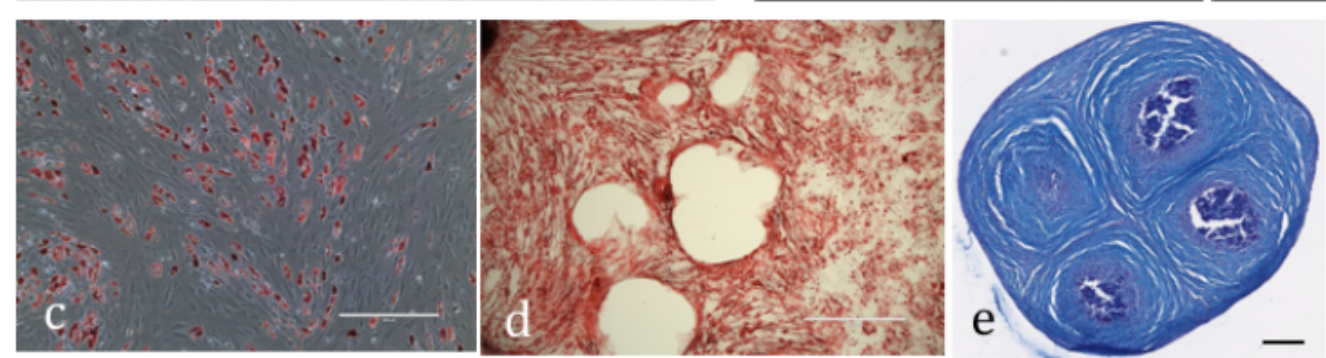

b

Figure 1: (a) ASCs in culture (b) Flow cytometry marker (panel) results for adipose derived cells. $\mathrm{N} \geq 3$. Adipose derived stem cells differentiated into; (c) adipocytes showing Oil Red O staining scale bar $=400 \mu \mathrm{m}$, (d) osteocytes showing Van Kossa staining scale bar=1mm, and (e) chondrocytes showing Alcian Blue staining. Scale bar $=500 \mu \mathrm{m}$. 
Citation: Coronado RE, Somaraki-Cormier M, Christy R, Natesan S, Ong J, et al. (2018) Chemical Induction of Human Adipose Stromal Cells Into Hepatocyte-Like Cells under Various Differentiation Conditions. J Stem Cell Res Ther 8: 424. doi: 10.4172/2157-7633.1000424

Page 5 of 10

\begin{tabular}{|c|c|c|c|c|}
\hline Condition & Pre-Induction (48hrs) & Induction (48hrs) & Differentiation (7days) & Maturation (7days) \\
\hline \multicolumn{5}{|c|}{ DMEM, Low-glucose } \\
\hline A & DMEM, Low-glucose & EGF+bFGF+Activin A & \multirow{2}{*}{$\begin{array}{c}\text { HGF+bFGF+FGF4+ } \\
\text { Nicotinamide+5\% } \\
\text { Serum }\end{array}$} & \multirow{2}{*}{$\begin{array}{l}\text { Oncostatin M+ITS+Dexamethasone } \\
+2 \% \text { Serum }\end{array}$} \\
\hline B & DMEM, Low-glucose & $\mathrm{EGF}+\mathrm{bFGF}+\mathrm{FGF} 4$ & & \\
\hline Condition & Pre-Induction (48hrs) & Induction (48hrs) & Differentiation (7days) & Maturation (7days) \\
\hline Control & \multicolumn{4}{|c|}{ DMEM, Low-glucose , $2 \%$ Serum } \\
\hline C & DMEM, Low-glucose & FGF4 & \multirow{3}{*}{ HGF } & HGF+ITSPre+Dex+OncM \\
\hline $\mathrm{D}$ & DMEM, Low-glucose & FGF4 & & HGF+ITSPre+Dex+TSA \\
\hline$E$ & DMEM, Low-glucose & FGF4 & & Palmitoyl+ITSPre+Dex+TSA \\
\hline
\end{tabular}

Table 1: Various methods for the differentiation of ASCs into hepatocyte-like cells
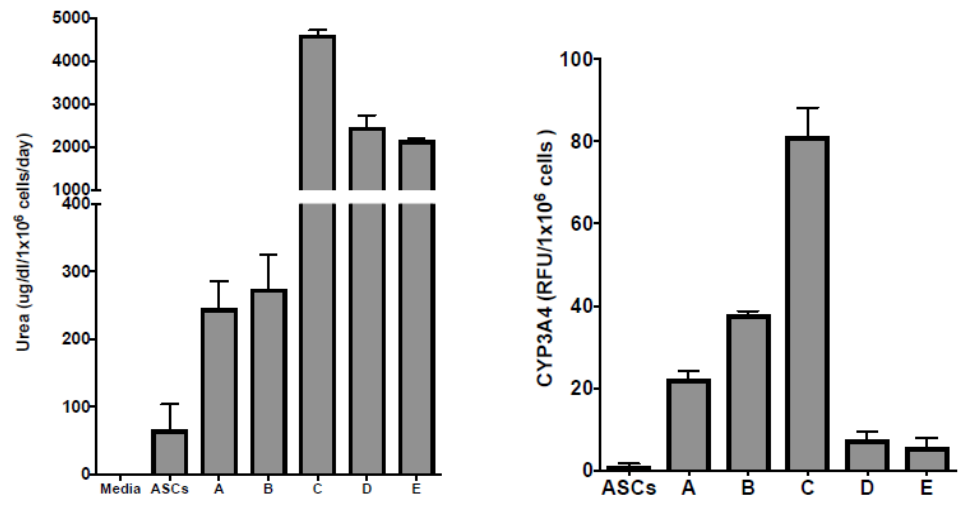

Figure 2: (a) Urea production per unit number cells by ASCs and Hepatocyte-like cells under different differentiation conditions. (b) CYP3A4 activity by ASCs and hepatocyte-like cells under different conditions Data is expressed as the means \pm SD of independent measurements.
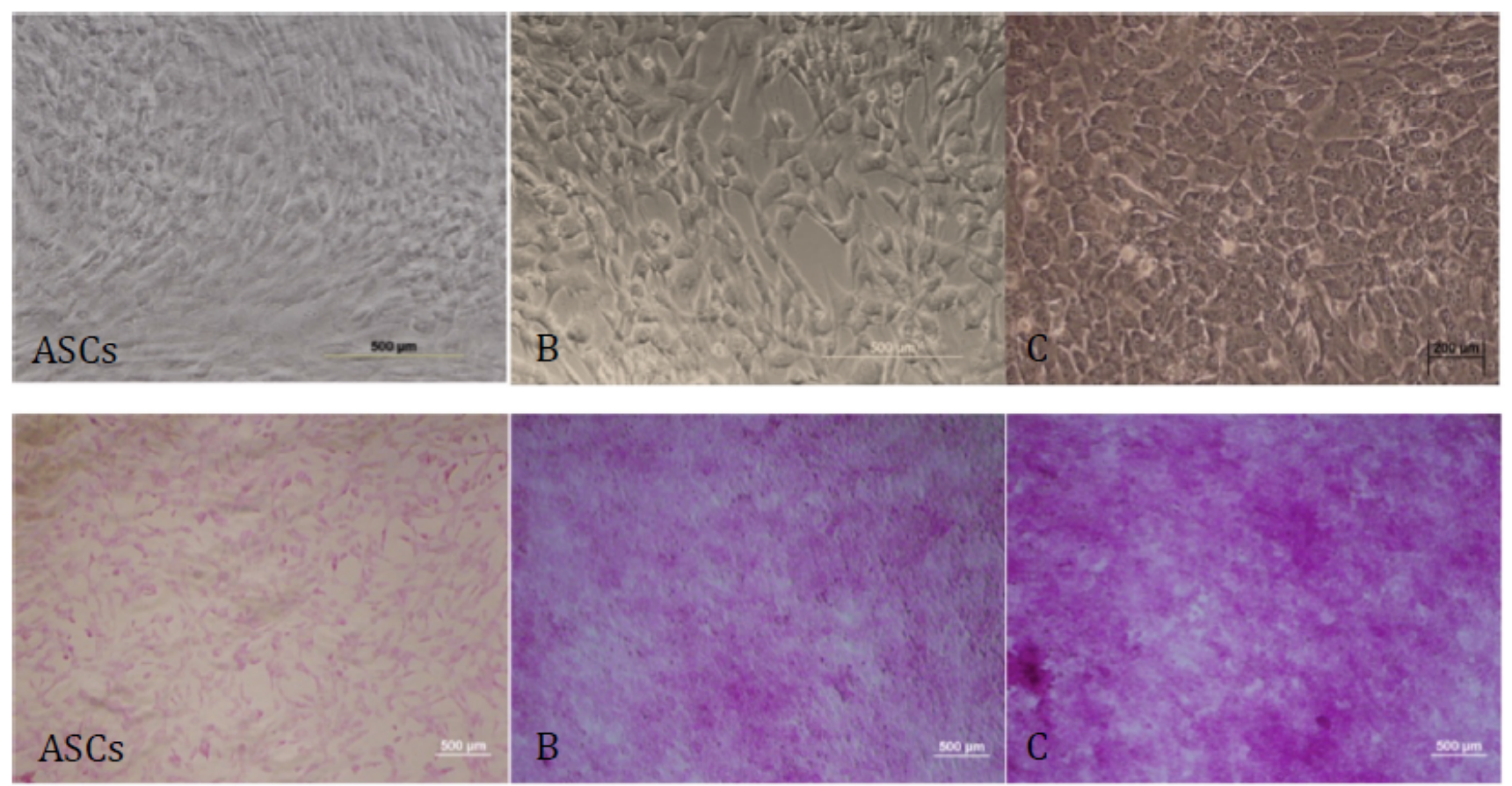

Figure 3: Periodic Acid-Schiff (PAS) staining (bottom images) on adipose derived mesenchymal stem cells differentiated into hepatocyte-like cells using protocol Condition B and Condition C. Top images are bright-field images displaying morphological changes. Bottom images are PAS stained cells. Scale bar $=500 \mu \mathrm{m}$. 
Citation: Coronado RE, Somaraki-Cormier M, Christy R, Natesan S, Ong J, et al. (2018) Chemical Induction of Human Adipose Stromal Cells Into Hepatocyte-Like Cells under Various Differentiation Conditions. J Stem Cell Res Ther 8: 424. doi: 10.4172/2157-7633.1000424

\section{Cytochrome P450 (C3A4) activity in hepatocyte-like cells}

Cytochrome P450 activity is a highly hepatocyte-specific function and is one of the main enzymes responsible for drug metabolism. ASCs differentiated under condition $\mathrm{C}$ and condition $\mathrm{B}$, displayed the highest values for cytochrome P450 (C3A4) activity (Figure 2b).

\section{Ability of Hepatocyte-like cells to store glycogen and LDL Uptake}

PAS staining detected the highest glycogen storage under condition C (Figure 3). In addition, the differentiated ASCs under condition C displayed the greatest degree of morphological change, from spindle
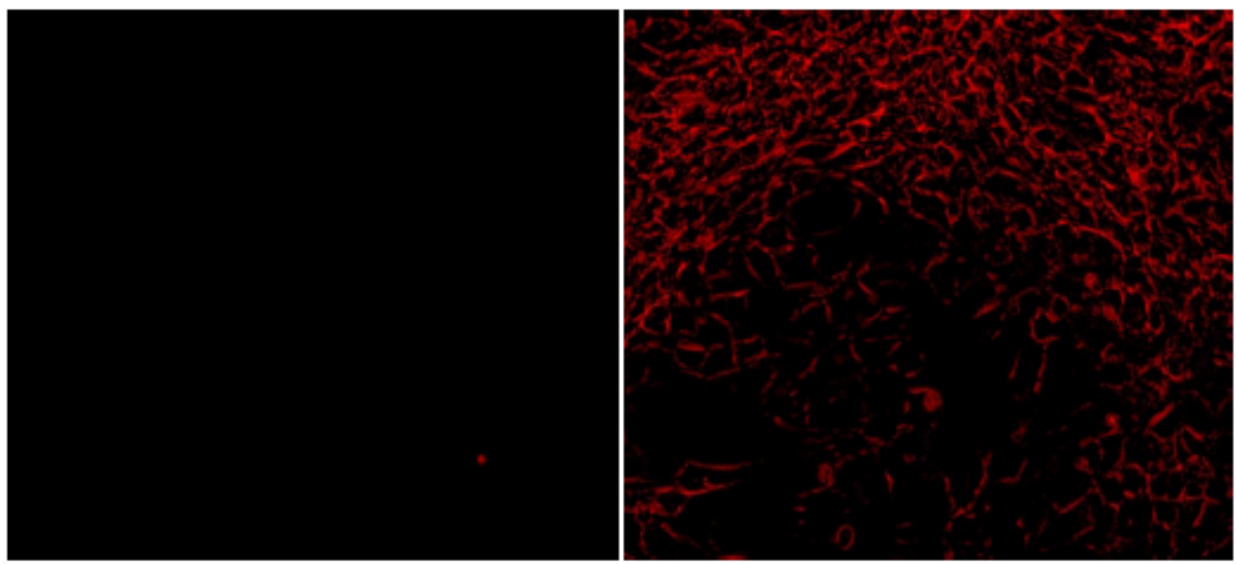

Figure 4: Low-density lipoprotein (LDL) staining on adipose derived mesenchymal stem cells differentiated into hepatocytelike cells using protocol Condition C (Right) and its respective non-differentiated control (Left) 100X.
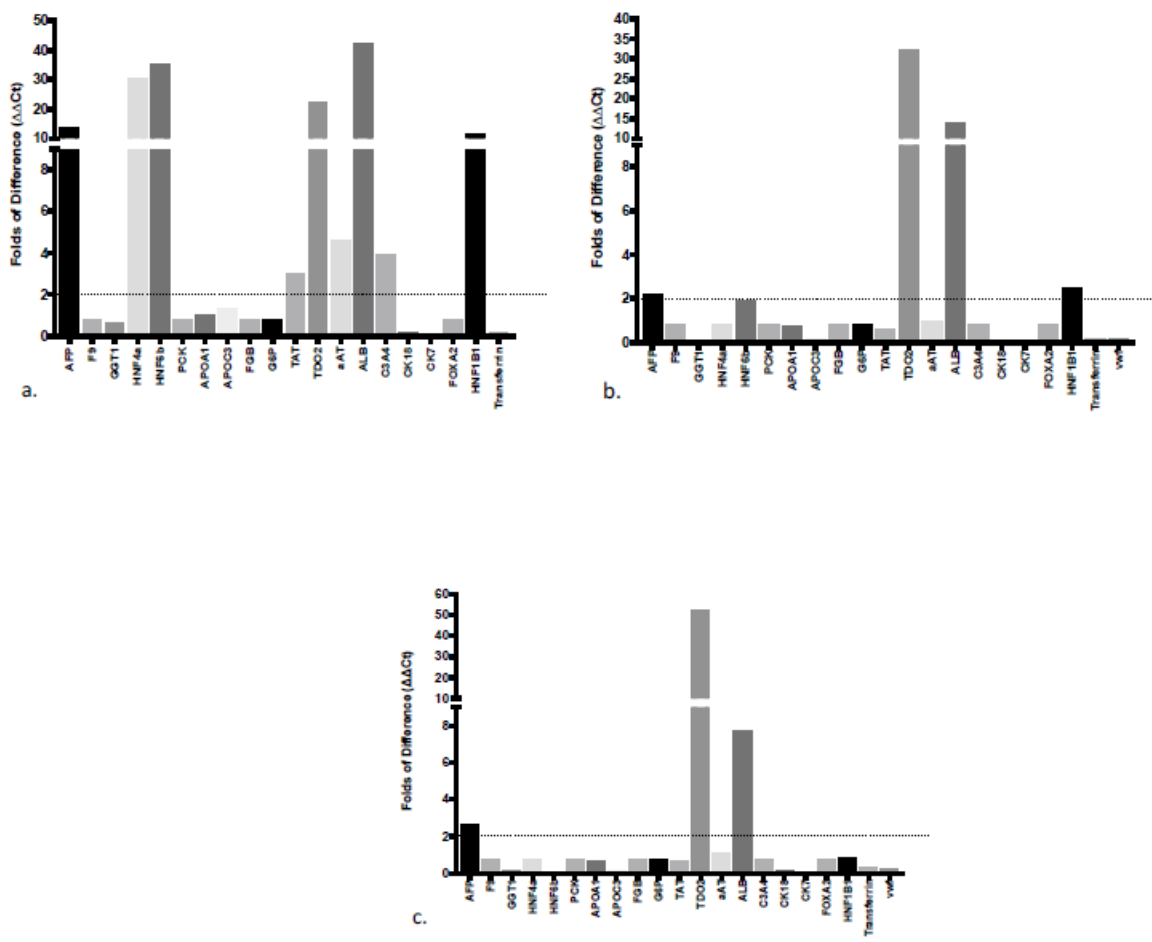

Figure 5: Hepatocyte-related gene expression of adipose derived mesenchymal stem cells after being differentiated into hepatocytelike cells using (a) Condition $C$ (b) Condition $A$, and (c) Condition B. Fold changes $(\Delta \Delta \mathrm{Ct})$ higher than 2 were considered upregulated, indicated by the dotted horizontal line. Abbreviations: AFP=Alphafetoprotein; F9=Coagulation Factor IX; GGT1=Gglutamyltranspeptidase; HNF6b=Hepatocyte Nuclear Factor 6b; PCK=phosphoenolpyruvate carboxykinase; APOA1=apolipoprotein A-I; APOC3=apolipoprotein C-III; FGB=fibrinogen beta chain; TAT=tyrosine aminotransferase; TDO2=tryptophan-2,3 dioxygenase; aAt=a-antitrypsin; ALB, albumin; C3A4=cytochrome P450/3A4; CK18, cytokeratin 18; CK7, cytokeratin 7; FOXA2=forkhead box protein A2; HNF1B1=Hepatocyte Nuclear Factor 1 B1; Transferrin=Transferrin. 
Citation: Coronado RE, Somaraki-Cormier M, Christy R, Natesan S, Ong J, et al. (2018) Chemical Induction of Human Adipose Stromal Cells Into Hepatocyte-Like Cells under Various Differentiation Conditions. J Stem Cell Res Ther 8: 424. doi: 10.4172/2157-7633.1000424

Page 7 of 10

cell type to a more "cuboidal" phenotype compared to condition B (Figure 3). Lastly, the ability of hepatocyte-like cells to uptake LDLs, a cardinal characteristic of true hepatocytes to clear LDLs from the blood, was evaluated by fluorescence microscopy. The results displayed that hepatocyte-like cells incorporated significantly more Dil-LDL that ASCs in culture (Figure 4).

\section{Hepatocyte-like gene expression analysis}

Comparison of gene expression of the hepatocyte-like cells and non-differentiated ACSs across the three treatments. The 21 genes we examined are drug metabolism related genes (drug transporter, Phase I metabolizing enzymes, Phase II Metabolizing, and Mesoderm) and
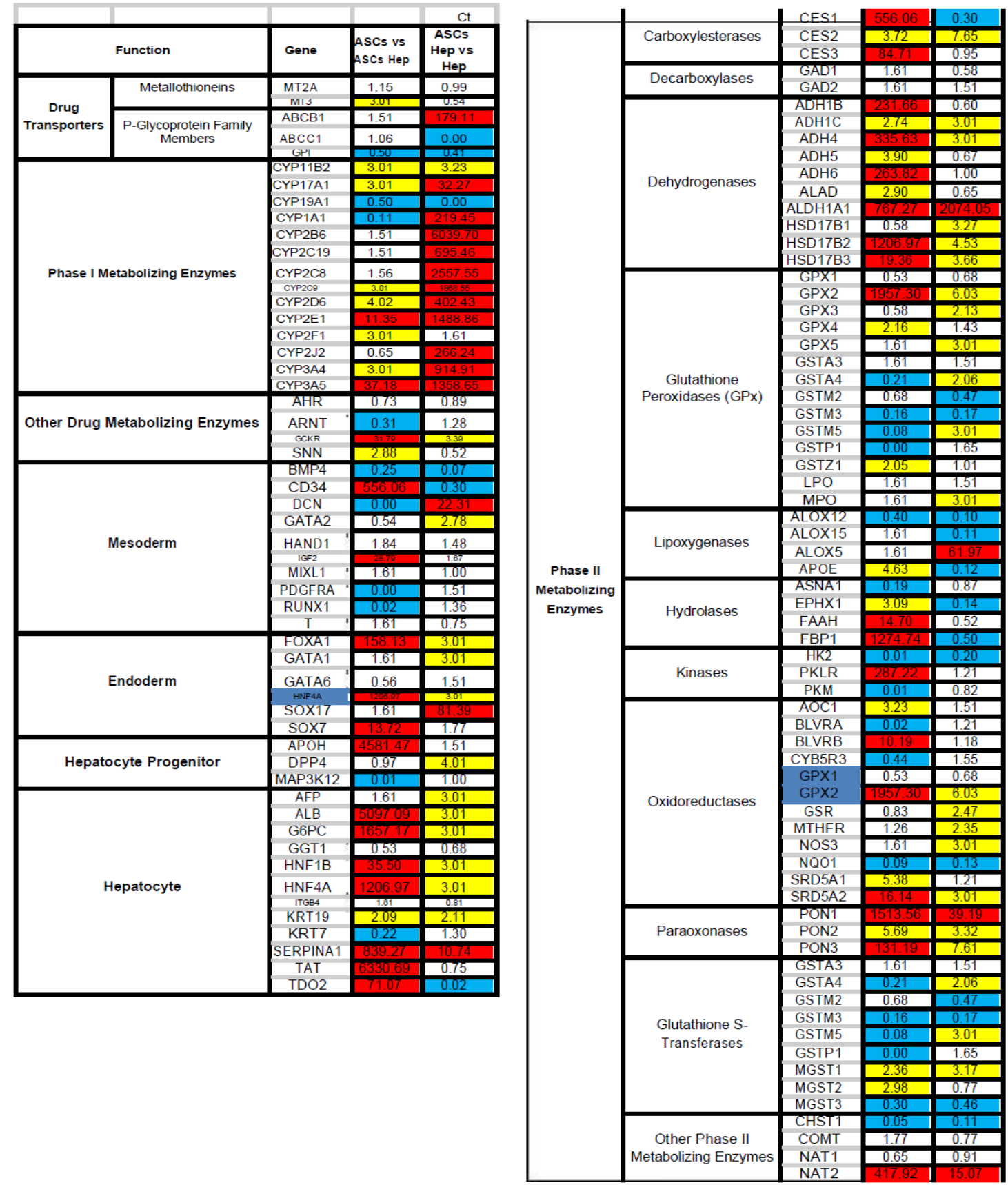

Table 2: Gene expression of hepatocyte -like cells (ASCs Hep) compared to undifferentiated ASCs (first column) and hepatocyte-like cells (ASCs Hep) compared to primary hepatocytes (second column) 
developmental genes (endoderm, mesoderm, Hepatocyte progenitor, and hepatocytes). All three treatments resulted in increased expression of Alphafetoprotein (AFP), tryptophan-2,3-dioxygenase (TDO2), and albumin (ALB). However, the highest number of up-regulated genes corresponding to mature hepatocyte functions was 9 (of 21), observed with condition $\mathrm{C}$ (Figure 5a). More comprehensive gene analysis comparing our protocol $\mathrm{C}$ to differentiate ASCs to hepatocyte-like cells resulted in the upregulation of 50 hepatocyte-related genes compared to non-differentiated ASCs (Table 2- First column ASCs vs. ASCs Hep). With the highest ( $>10$ fold) up-regulation detected in the following genes: CYP2E1, CYP3A5, ALDH1A1, ALOX5, PON1, NAT2, DCN, SOX17, SERPINA1. Followed by genes showing upregulation $(>2$ fold and <10 fold): MT3, CYP11B2, CYP17A1, CYP2C9, CYP2D6, CYP2F1, CYP3A4, CES2, ADH1C, ADH4, HSD17B1, HSD17B2, HSD17B3, GPX2, GPX3, GPX5, GSTA4, GSTM5, MPO, GSR, MTHFR, NOS3, SRD5A2, PON2, PON3, GSTA4, GSTM5, MGST1, GCKR, GATA2, FOXA1, GATA1, DPP4, AFP, ALB, G6PC, HNF1B, HNF4A, KRT19. Furthermore, when differentiated ASCs into hepatocytes-like cells where compared to primary hepatocytes, primary hepatocytes showed upregulation of 60 hepatocyte-related genes (Table 2- Second column ASCs Hep vs. Hep). With the highest ( $>10$ fold) up-regulation detected in the following genes: ABCB1, CYP17A1, CYP1A1, CYP2B6, CYP2C19, CYP2C8, CYP2C9, CYP2D6, CYP2E1, CYP2J2, CYP3A4, CYP3A5, CES1, CES3, ADH1B, ADH4, ADH6, ALDH1A1, HSD17B2, HSD17B3, GPX2, FAAH, FBP1, PKLR, BLVRB, SRD5A2, PON1, PON3, NAT2, GCKR, CD34, IGF2, FOXA1, HNF4A, SOX7, APOH, ALB, G6PC, HNF1B, SERPINA1, TAT, TDO2. Followed by genes showing upregulation ( $>2$ fold and $<10$ fold): CYP11B2, CES2, ADH1C, ADH5, ALAD, GPX4, GSTZ1, APOE, EPHX1, AOC1, SRD5A1, PON2, MGST1, MGST2, SNN, KRT19.

Even though hepatocyte-like ASCs expression of hepatocyte-related genes was much inferior than those of primary hepatocyte like cells, some genes in hepatocyte like -ASCs cells were higher (upregulated) when compared to primary hepatocytes, mainly those related to Glutathione S-Transferase functions (Table 2).

\section{Discussion}

Human ASCs have been in the spotlight of regenerative medicine for a long time. Primarily, because adipose tissue is readily available, they are acquired with minimally invasive surgery, can be easily isolated, cultured in vitro, and have a broad differentiation potential including tri-lineage differentiation into mesodermal lineage cells like: osteocytes, chondrocytes, and adipocytes. More recently, there have been many studies attempting to differentiate ASCs into hepatocytelike cells, with the intention of replacing the need of primary hepatocytes and use them for clinical applications related to liver diseases. Some of the investigated hepatocyte differentiation methods require long time (months) to induce differentiation and some even included vector-integrated transcription factors to reprogram ASCs into hepatocyte-like cells rendering the later unsafe to recipient [48]. In order to study the differentiation of ASCs into hepatocyte-like cells, we compared different methods of differentiation and evaluated three key hepatocyte-related functions: ureagenesis, P450 metabolism, and glycogen storage. Ureagenesis from ammonia occurs exclusively in the liver and involves both cytosolic and mitochondrial reactions and is a valuable global indicator of hepatic performance [49]. Cytochrome P450 (C3A4) metabolism is a functional process that represents the capability to biotransform xenobiotics, which is one of the most relevant characteristics of mature hepatocytes.

Therefore, there is a great need to ensure the appropriate metabolic performance of cells when cultured in vitro. Cytochrome P450 enzymes are essential for the metabolism of many medications. Although this class has more than 50 enzymes, six of them metabolize 90 percent of drugs, with the two most significant enzymes being CYP3A4 and CYP2D6 [50]. Glycogen storage/glucogenesis is a central function performed by mature hepatocytes in the liver and is an essential mechanism in survival because it provides readily available glucose in order to supply the tissues with an oxidizable energy source [51,52].

In addition to glycogen storage, hepatocytes play a protagonist role in cholesterol homeostasis, critical for normal physiological functions. Hepatocytes are responsible for cellular cholesterol metabolism and clearance of plasma cholesterol. Hepatocyte mediated cholesterol metabolism is dependent on hepatocyte LDL receptor mediated uptake, rendering the evaluation of LDL uptake a function of great significance [53]. Although these functions (Urea, P450, glycogen storage, morphology and LDL uptake) and 9 genes do not completely cover all functions of hepatocytes, they are considered reliable functional assays to show hepatocyte specificity [24,27,28]. Moreover, these are rational and practical elements to compare the hepatocyte nature of differentiated cells. It is important to clarify that even the highest values of our hepatocyte-like obtained for urea, glycogen, and P450 metabolism are not necessarily comparable to those values found of mature primary hepatocytes. However, obtaining the highest values possible from all protocols can provide information about the hepatocyte-functional limit conditional to the stem cell nature. The same stands for gene expression analysis of ASC-derived hepatocytelike cells which demonstrated an average upregulation of 47 hepatocyte related genes after hepatocyte differentiation (Table 2) compared to the original state of the cells. Some generalized trends can be seen by comparing the two groups. First, it is clear that in Phase 1 metabolic enzymes, the expression levels in the primary hepatocytes are much greater than in the differentiated ASCs (see the predominance of red cells in Table 2 on the second column). This trend is reversed in Endoderm, Hepatocytes, and Dehydrogenases comparisons, with more red cells in the first column (ASCs differentiated to hepatocyte-like cells).

The protocols used in this study were carefully chosen from published literature with the intention to consolidate, with minor modifications, the most common strategies utilized to differentiate ASCs into hepatocyte-like cells in order to elucidate which methodology could be more appropriate to generate hepatocyte-like cells $[27,28,30]$.

Although the functional values of hepatocyte-like cells acquired with the different protocols were not as high as those of primary hepatocytes, protocol $\mathrm{C}$ (Table 1) produced the highest values. We hypothesize the combination of Oncostatin M (oncM) and HGF in the presence of Dex in protocol C, not included in the other protocols, is responsible for the higher functional values and better hepatocyte-like cell morphology. OncM is a cytokine that is involved in hepatocyte development mediated through the STAT3 pathway during the fetal stages of growth. In contrast, HGF is upregulated after birth and plays an important role in hepatocyte development and maturation through a STAT3-independent pathway, not yet elucidated. It has been shown that the combination of OncM and HGF can induce hepatocyte maturation in the presence of Dex in vitro [54].

Though ASCs may not be the best candidates to generate high quality hepatocyte-like cells they are not the only stromal cells available. We are currently in process of studying various stromal cells from different tissue origins in hope to find the best cell type to generate hepatocyte-like cells. 
Citation: Coronado RE, Somaraki-Cormier M, Christy R, Natesan S, Ong J, et al. (2018) Chemical Induction of Human Adipose Stromal Cells Into Hepatocyte-Like Cells under Various Differentiation Conditions. J Stem Cell Res Ther 8: 424. doi: 10.4172/2157-7633.1000424

Page 9 of 10

The above results may be discouraging at first, but looking closer, there are some genes that were similar (between -2 and 2 folds) or even higher to those of primary hepatocytes. Although, ASCderived hepatocyte-like cells were not comparable overall to primary hepatocytes, some specific genes were comparable or even superior than those of hepatocytes, for example those relevant to Phase II metabolism genes family: CYP19A1, glutathione peroxidase gene family (GSTA4, GSTM3, GSTM5, GSTP1, kinases (HK2, PKM), which could represent an opportunity to identify which cases these cells could provide therapeutic benefits for some of the most troublesome liver pathologies.

\section{References}

1. Moreno R, Berenguer M (2006) Post-liver transplantation medical complications. Ann Hepatol 5: 77-85. [PubMed]

2. Roberts MS (2004) Survival after liver transplantation in the United States: A disease-specific analysis of the UNOS database. Liver Transplantation 10: 886-897. [PubMed]

3. Lucey MR (1998) Minimal criteria for placement of adults on the liver transplant waiting list: a report of a national conference organized by the American Society of Transplant Physicians and the American Association for the Study of Liver Diseases. Transplantation 66: 956-962.

4. Baccarani U (2005) Human hepatocyte transplantation for acute liver failure: state of the art and analysis of cell sources. Transplant Proc 37: 2702-2704. [PubMed]

5. Fisher RA, Strom SC (2006) Human hepatocyte transplantation: worldwide results. Transplant 82: 441-449.

6. Wang F (2014) Monitoring of intrasplenic hepatocyte transplantation for acuteon-chronic liver failure: a prospective five-year follow-up study. Transplant Proc 46: 192-198. [PubMed]

7. Kim WH (2000) Systematic analysis of the effects of hepatocyte transplantation on rats with acute liver failure. Hepatogastroent 47: 371-374.

8. Enns GM, Millan GT (2008) Cell-based therapies for metabolic liver disease. Mol Genet Metab 95: 3-10. [PubMed]

9. Nussler A (2006) Present status and perspectives of cell-based therapies for liver diseases. J Hepatol 45: 144-159.

10. Dianat, N (2013) Human pluripotent stem cells for modelling human liver diseases and cell therapy. Curr Gene Ther 13: 120-132. [PubMed]

11. Touboul T (2010) Generation of functional hepatocytes from human embryonic stem cells under chemically defined conditions that recapitulate liver development. Hepat 51: 1754-1765. [PubMed]

12. Si-Tayeb K (2010) Highly Efficient Generation of Human Hepatocyte-Like Cells from Induced Pluripotent Stem Cells. Hepat 51: 297-305.

13. Zemel R (2009) Expression of liver-specific markers in naive adipose-derived mesenchymal stem cells. Liver Int 29: 1326-1337. [PubMed]

14. Sancho-Bru P (2011) Therapeutic possibilities of stem cells in the treatment of liver diseases. Gastroenterol Hepatol 34: 701-710.

15. Pilegg A (2013) Mesenchymal stromal (stem) cells to improve solid organ transplant outcome: lessons from the initial clinical trials. Curr Opin Organ Transplant 18:672-681. [PubMed]

16. Obermajer N (2014) Rationale and prospects of mesenchymal stem cell therapy for liver transplantation. Curr Opin Organ Transplant 19: 60-64.

17. Amariglio N (2009) Donor-derived brain tumor following neural stem cell transplantation in an ataxia telangiectasia patient. PLoS Med 6: e1000029. [PubMed]

18. Miura K (2009) Variation in the safety of induced pluripotent stem cell lines. Nat Biotechnol 27: 743-745.

19. Doi D (2012) Prolonged maturation culture favors a reduction in the tumorigenicity and the dopaminergic function of human ESC-derived neural cells in a primate model of Parkinson's disease. Stem Cells 30: 935-945. [PubMed]
20. Lee AS (2013) Tumorigenicity as a clinical hurdle for pluripotent stem cell therapies. Nat Med 19: 998-1004

21. Natesan S (2011) Debrided skin as a source of autologous stem cells for wound repair. Stem Cells 29: 1219-1230. [PubMed]

22. Shi XL (2005) Hepatocyte-like cells from directed differentiation of mouse bone marrow cells in vitro. Acta Pharmacol Sin 26: 469-476.

23. Ilic Z, Leffert H, Sell S (2006) Sequential exposure to cytokines reflecting embryogenesis: The key for in vitro differentiation of adult bone marrow stem cells into functional hepatocyte-like cells. Toxicol Sci 94: 235-239. [PubMed]24. Banas A (2007) Adipose tissue-derived mesenchymal stem cells as a source of human hepatocytes. Hepatology 46: 219-228.

24. Snykers S (2007) Chromatin remodeling agent trichostatin A: a key-factor in the hepatic differentiation of human mesenchymal stem cells derived of adult bone marrow. BMC Dev Biol 7: 24. [PubMed]

25. Yamamoto $Y$ (2008) A comparative analysis of the transcriptome and signal pathways in hepatic differentiation of human adipose mesenchymal stem cells. FEBS J 275: 1260-1273.

26. Aurich $\mathrm{H}$ (2009) Hepatocyte differentiation of mesenchymal stem cells from human adipose tissue in vitro promotes hepatic integration in vivo. Gut 58:570581. [PubMed]

27. Banas A (2009) Rapid hepatic fate specification of adipose-derived stem cells and their therapeutic potential for liver failure. J Gastroenterol Hepato 24: 70-77.

28. Ishikawa T (2010) Stem cells for hepatic regeneration: the role of adipose tissue derived mesenchymal stem cells. Curr Stem Cell Res Ther 5: 182-189. [PubMed]

29. Lue J (2010) Transdifferentiation of adipose-derived stem cells into hepatocytes: a new approach. Liver Int 30: 913-22.

30. Ruiz JC (2010) Differentiated Human Adipose-Derived Stem Cells Exhibit Hepatogenic Capability In Vitro and In Vivo. J Cell Physiol 225: 429-436. [PubMed]

31. Gómez-Lechón MJ (1996) Isolation, Culture and Use of Human Hepatocytes in Drug Research, in In Vitro Methods in Pharmaceutical Research, J.V. Castell and M.J. Gómez-Lechón, Editors. 1996, Academic Press: San Diego: 129-153.

32. LeCluyse EL (2001) Human hepatocyte culture systems for the in vitro evaluation of cytochrome P450 expression and regulation. Eur J Pharm Sci 13: 343-368. [PubMed]

33. Bissell DM (1987) Support of cultured hepatocytes by a laminin-rich gel. Evidence for a functionally significant subendothelial matrix in normal rat liver. J Clin Invest 79: 801-812. [PubMed]

34. Wang YJ (2004) Primary hepatocyte culture in collagen gel mixture and collagen sandwich. World J Gastroenterol 10: 699-702. [PubMed]

35. Fassett J, Tobolt D (2006) Type I collagen structure regulates cell morphology and EGF signaling in primary rat hepatocytes through cAMP-dependent protein kinase A. Mol Biol Cell 17: 345-356. [PubMed]

36. Zeisberg M (2006) De-differentiation of primary human hepatocytes depends on the composition of specialized liver basement membrane. Mol Cell Biochem 283: 181-189. [PubMed]

37. Plant AL (2009) Cell response to matrix mechanics: focus on collagen. Biochim Biophys Acta 1793: 893-902.

38. Zhang Y (2009) Tissue-specific extracellular matrix coatings for the promotion of cell proliferation and maintenance of cell phenotype. Biomater 30: 4021 4028. [PubMed]

39. te Velde AA (1995) Functional activity of isolated pig hepatocytes attached to different extracellular matrix substrates. Implication for application of pig hepatocytes in a bioartificial liver. J Hepatol 23: 184-192. [PubMed]

40. Hamilton GA (2001) Regulation of cell morphology and cytochrome P450 expression in human hepatocytes by extracellular matrix and cell-cell interactions. Cell Tissue Res 306: 85-99. 
Citation: Coronado RE, Somaraki-Cormier M, Christy R, Natesan S, Ong J, et al. (2018) Chemical Induction of Human Adipose Stromal Cells Into Hepatocyte-Like Cells under Various Differentiation Conditions. J Stem Cell Res Ther 8: 424. doi: 10.4172/2157-7633.1000424

Page 10 of 10

41. Mooney D (1992) Switching from differentiation to growth in hepatocytes: control by extracellular matrix.

42. J Cell Physiol 151: p. 497-505. [PubMed]

43. Lorenti A (2001) Culture of porcine hepatocytes: the dogma of exogenous matrix revisited. Artif Organs 25: 546-550. [PubMed]

44. Du Y (2006) 3D hepatocyte monolayer on hybrid RGD/galactose substratum. Biomater 27: 5669-5680. [PubMed]

45. Mitaka T (1999) Reconstruction of hepatic organoid by rat small hepatocytes and hepatic nonparenchymal cells. Hepatology 29: 111-125. [PubMed]

46. LeCluyse E (1999) Influence of extracellular matrix overlay and medium formulation on the induction of cytochrome $\mathrm{P}-4502 \mathrm{~B}$ enzymes in primary cultures of rat hepatocytes. Drug Metab Dispos 27: 909-915.

47. Dominici M (2006) Minimal criteria for defining multipotent mesenchymal stromal cells. The International Society for Cellular Therapy position statement. Cytotherapy 8: 315-317. [PubMed]
48. Fu Y (2016) Rapid generation of functional hepatocyte-like cells from human adipose-derived stem cells. Stem Cell Res Ther 7: 105. [PubMed]

49. Watford M (1991) The urea cycle: a two-compartment system. Essays Biochem 26: 49-58. [PubMed]

50. Lynch T (2007) The effect of cytochrome P450 metabolism on drug response interactions, and adverse effects. Am Fam Physician 76: 391-396. [PubMed]

51. Bechmann LP (2012) The interaction of hepatic lipid and glucose metabolism in liver diseases. J Hepatol, 56: 952-964. [PubMed]

52. Berg JM (2002) Glycogen Metabolism in Biochemistry, New York: W H Freeman. [PubMed]

53. Edge SB (1986) Evidence for metabolism of low density lipoproteins by a pathway independent of the classical low density lipoprotein receptor. $\mathrm{J}$ Bio Chem 261: 3800-3806. [PubMed]

54. Kamiya AT (2001) Oncostatin M and hepatocyte growth factor induce hepatic maturation via distinct signaling pathways. FEBS Lett 492: 90-94. [PubMed] 